\title{
Opportunities and Challenges in OFDMA-Based Cellular Relay Networks: A Radio Resource Management Perspective
}

\author{
Mohamed Salem, Student Member, IEEE, Abdulkareem Adinoyi, Member, IEEE, \\ Halim Yanikomeroglu, Member, IEEE, and David Falconer, Life Fellow, IEEE
}

\begin{abstract}
The opportunities and flexibility in relay networks and orthogonal frequency-division multiple access (OFDMA) make the combination a suitable candidate network and air-interface technology for providing reliable and ubiquitous high-data-rate coverage in next-generation cellular networks. Advanced and intelligent radio resource management (RRM) schemes are known to be crucial toward harnessing these opportunities in future OFDMA-based relay-enhanced cellular networks. However, it is not very clear how to address the new RRM challenges (such as enabling distributed algorithms, intra-cell/inter-cell routing, intense and dynamic co-channel interference (CCI), and feedback overhead) in such complex environments comprising a plethora of relay stations (RSs) of different functionalities and characteristics. Employment of conventional RRM schemes in such networks will highly be inefficient if not infeasible. The next-generation networks are required to meet the expectations of all wireless users, irrespective of their locations. High-data-rate connectivity, mobility, and reliability, among other features, are examples of these expectations. Therefore, fairness is a critical performance aspect that has to be taken into account in the design of prospective RRM schemes. This paper reviews some of the prominent challenges involved in migrating from the conventional cellular architecture to the relay-based type and discusses how intelligent RRM schemes can exploit the opportunities in relay-enhanced OFDMA-based cellular networks. We identify the role of multiantenna systems and explore the current approaches in literature to extend the conventional schedulers to next-generation relay networks. This paper also highlights the fairness aspect in such networks in the light of the recent literature, provides some example fairness metrics, and compares the performances of some representative algorithms.
\end{abstract}

Index Terms-Cellular, fairness, load balancing, orthogonal frequency-division multiple access (OFDMA), radio resource management (RRM), relaying, routing, scheduling, throughput.

\section{INTRODUCTION}

$\mathbf{U}$ BIQUITOUS high-data-rate coverage is the theme of next-generation wireless networks. Given the expen-

Manuscript received April 3, 2009; revised September 30, 2009; accepted January 8, 2010. Date of publication February 17, 2010; date of current version June 16, 2010. This work was supported by Samsung Electronics Company Ltd., Samsung Advanced Institute of Technology, Korea. The review of this paper was coordinated by Dr. W. Zhuang.

M. Salem, H. Yanikomeroglu, and D. Falconer are with the Department of Systems and Computer Engineering, Carleton University, Ottawa, ON K1S 5B6, Canada (e-mail: mrashad@sce.carleton.ca; halim@sce.carleton.ca; ddf@sce.carleton.ca).

A. Adinoyi is with Swedtel Arabia, Riyadh 11527, Saudi Arabia (e-mail: adinoyi@sce.carleton.ca).

Color versions of one or more of the figures in this paper are available online at http://ieeexplore.ieee.org.

Digital Object Identifier 10.1109/TVT.2010.2042736 sive and scarce spectrum, achieving this objective requires high-spectral-efficiency schemes that rely on aggressive resource reuse. Meanwhile, the orthogonal frequency-division multiplexing (OFDM) air interface is the accepted candidate technology for delivering this ambitious performance for nextgeneration networks. This is mainly due to the fact that OFDM has the inherent ability to combat frequency-selective fading. In addition, it offers some degrees of freedom in radio resource management (RRM) since orthogonal frequency-division multiple access (OFDMA)-based RRM schemes allocate different portions of radio resources to different users in both the frequency and time domains.

Ubiquitous coverage demands that service has to reach users in the most unfavorable channel conditions (e.g., cell-edge users) by efficient distribution of the high data rate (capacity) across the network. Increasing capacity along with coverage in conventional cellular architecture (without relay assistance) dictates dense deployment of base stations (BSs), which turns out to be a cost-wise inefficient solution to service providers [1]. A relay station (RS) with less cost and functionality than the BS is able to extend the high-data-rate coverage to remote areas in the cell. In addition to the traditional use of relays to enhance network coverage, a new network architecture representing a paradigm shift has recently emerged. This architecture is widely referred to as cooperative communications. Protocols to facilitate relay cooperation are still being investigated. Thus, numerous standardization bodies, forums, and consortiums have been positioning their efforts toward relaybased architectures in various scenarios, e.g., IEEE $802.16 \mathrm{~m}$ and Third-Generation Partnership Project advanced long-term evolution (LTE-Advanced).

OFDMA combined with relaying techniques offers a promising technology for providing ubiquitous high-data-rate coverage. While it is crucial to devise intelligent RRM schemes to harness the opportunities in future relay-enhanced OFDMAbased networks, it is not yet clear how to overcome the new challenges in such a complex environment where conventional RRM schemes are not scalable. In fact, even some of the conventional technical terms may need to be redefined. Nevertheless, with the ambitious objective of ubiquitous high-data-rate coverage announced and the opportunities advertised, modern RRM schemes have stronger fairness obligations, and thus, the RRM design approach has to be shifted from network centric toward user centric [2], [3]. 


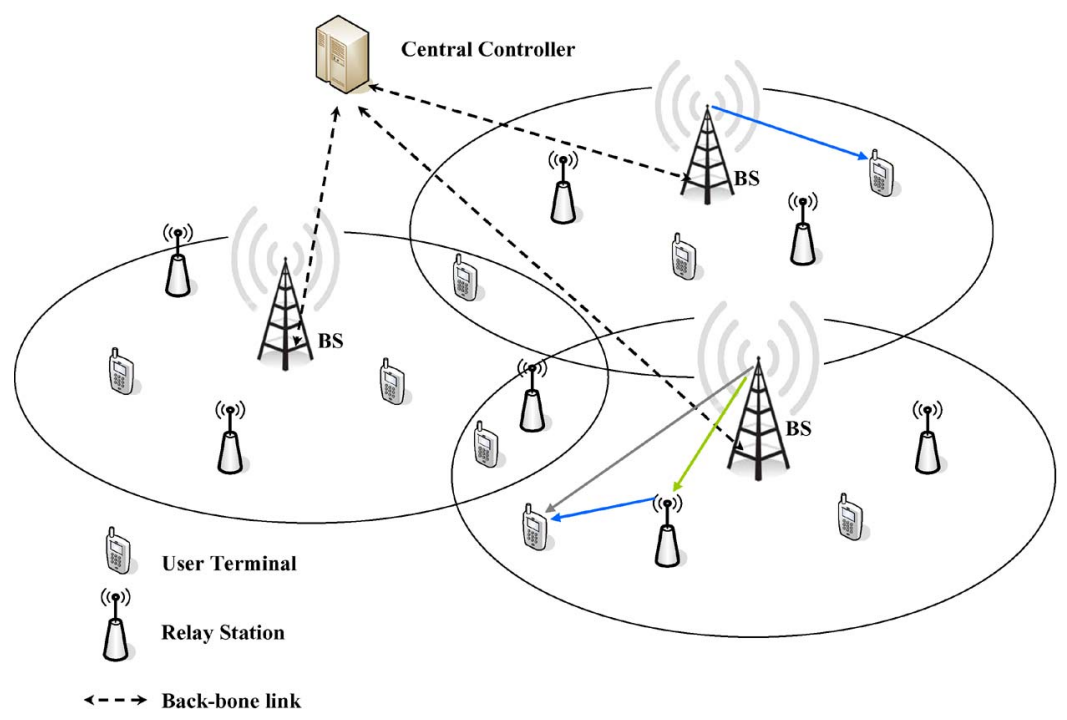

Fig. 1. Example relay-enhanced cellular architecture. A central controller performs global resource allocation in centralized conventional networks.

From an RRM perspective, in Section II, we address some of the opportunities, challenges, and terminologies associated with the migration from conventional cellular architecture to relay enhanced in OFDMA-based networks. Some of these issues are discussed in [4] yet within the limited depth and literature breadth of a magazine article. In Section V, we highlight the fairness concerns in such networks and discuss some fairness metrics as well as possible fairness implementations in radio resource allocation (RRA) algorithms. An interesting case study that combines these issues is discussed in Section VI, along with relevant performance results to further the illustration.

\section{Orthogonal Frequency-Division Multiple Access-Based Cellular Relay Networks}

Research interest is moving rapidly toward the relay-based network architecture. The focus of this paper is mostly on the potential opportunities and challenges in this type of network architecture. To be on the side of caution, it may be necessary to reexamine the technical terminologies used in conventional networks as they relate to this new paradigm shift, i.e., relayenhanced networks.

\section{A. Migration From Conventional Cellular to Relay-Enhanced}

1) Centralized or Distributed?: There seems to be no agreement in the literature on the use of the terms "centralized," "decentralized," and "distributed" as regards to the operation of RRM schemes. For instance, in the context of conventional cellular networks (e.g., [5]), an RRM scheme is considered to be centralized if there exists a central controller that gathers all the information and feedback required from all the BSs and performs global resource allocation, as shown in Fig. 1. Whereas an RRM scheme may be considered distributed if each cell individually performs its own resource allocation based on local information and perhaps aided with some inter-cell information. In the context of relay-enhanced cellular networks, however, the latter scheme is referred to as centralized given that the BS gathers information from the RSs and performs the resource allocation for itself and the RSs as well.
One way to avoid such ambiguity is to tie the description to the relevant network "hierarchy." For instance, a network-level distributed/cell-level centralized RRM scheme could refer to a scheme that does not rely on a central controller to perform global resource allocation, whereas each BS will individually handle the resource allocation for all the entities in the cell including RSs. Similarly, a network-level distributed/cell-level semi-distributed RRM scheme could refer to a similar scheme but with the provision that RSs can partially participate in the resource allocation. The importance of such precise terminology cannot be overemphasized in future networks, where several entities will be involved, in one way or another, in the realization of RRM schemes.

In addition to that, the ambiguity of the technical terminologies extends to an important aspect that is "load balancing."

2) Cell-Load Balancing: Load balancing is a function usually incorporated with the connection admission control (CAC) mechanisms in conventional cellular networks. In that context, the load balancing function refers to the hand over (hand off) of some users between adjacent cells to distribute the traffic load network-wide among BSs while maintaining the users' quality of service (QoS). Although the load balancing previously defined will be an integral part of any prospective RRM scheme in relay-enhanced networks, researchers often associate the term "load balancing" in relay networks with a different function that aims at evenly distributing the load among all nodes, cell-wide. The number of OFDM subcarriers handled by a node is often employed in the literature as a good estimate of the traffic load at that node [6]-[9]. A balanced traffic load reduces the packet processing delays at the regenerative relays.

One might refer to the first definition as network-load balancing, whereas the second definition might be referred to as cell-load balancing. In fact, cell-load balancing also has some implications on inter-cell interference (ICI) and fairness, which we highlight in Sections II-B and V, respectively. We will discuss the concept of network-load balancing in relay networks in Section II-A5.

3) Various Forms of Wireless Relays of Different Functionalities: It is envisaged that a plethora of RSs of different 
specifications, functionalities, and geographical densities will be part of the next-generation cellular network architecture. For instance, fixed RSs (FRSs) are assumed to be deployed with low density at strategic locations of the cell; possibly line-of-sight (LOS) communication with the BS is maintained, and off-thewall power is available. Worthy of emphasis is the plug-andplay type of relay known as nomadic RS (NRS), an idea that has been entrenched in the IEEE 802.16 standards [10], [11]. While mobile RSs (MRSs) are characterized by their mobility (e.g., rooftop-mounted vehicular devices), NRSs are technically stationary devices but portable and, like MRSs, are battery operated; much lower transmit power levels than those of FRSs are thus anticipated. In [12], motivational scenarios for using mobile multihop relaying are provided. Therein, NRSs can be deployed with high density to provide temporary coverage and capacity in an area where FRSs may not provide the required QoS. An example of temporary coverage areas could be, in general, where wireless connectivity is required for only a short period of time, such as in trade fairs and sporting events or in disaster-recovery situations. In addition, NRSs can be used to spread the capacity in a large building. In such case, NRSs will coexist with FRSs with potentially much better communication links to user terminals. Although FRSs are the most commonly considered in the literature, to the extent of our knowledge, no work so far, other than [13] and [14], has provided mechanisms for integrating autonomous NRSs into the cellular network or suggested the underlying RRM schemes to facilitate a smooth integration and coexistence with FRSs.

As the architecture of next-generation cellular networks becomes more sophisticated, i.e., comprising a plethora of active nodes, distributed RRM schemes with limited feedback, particularly if involving MRSs, become essential. Furthermore, in a network with a large number of relays, more often than not, orthogonal resources are required for multihop relaying purposes, and thus, a form of reuse is necessary. To facilitate this reuse and combat the resulting interference, intelligent RRM schemes are needed to balance between aggressive resource reuse and efficient management of the associated co-channel interference (CCI).

Given such modern architecture, prospective RRM schemes are quite diverse in terms of transmission protocols and optimization objectives. Among the fundamental questions these protocols have to answer are the following; which transmission mode (direct, multihop simple relaying, or multihop cooperative relaying) is optimal for a particular user and which relay node(s) should be incorporated in that mode? This is where efficient routing schemes come as an RRM design tool.

4) Relay Selection or In-Cell Routing: Routing is thus a key issue of networks that support multihop relaying through the deployment of dedicated relays, users' cooperative relaying, or protocols incorporating both. Routing can be viewed as the process of establishing efficient connectivity between nodes over multihop links allowing coverage extension, throughput, and fairness improvement. Since different routing schemes are expected to differently affect the system performance in terms of throughput, delays, and signaling overhead, several relayselection strategies and relaying criterion are employed in the RRM schemes as an initial step followed by scheduling user packets on the chosen path(s), e.g., [15]. However, performing joint routing and scheduling is known to produce superior performance results, as compared with decoupled scheduling and routing [16]. Therefore, differently expressed, resource allocation in such relay-enhanced networks is indeed a joint scheduling and routing problem. However, it is quite challenging to devise efficient RRM schemes that tackle the joint problem. The algorithm presented in the case study is a good example of a class of dynamic joint routing and scheduling strategies discussed in the literature [17], as based on the theory in [18] and as applied to OFDMA relay networks [19]. In such a strategy, $\mathrm{CCI}$ is depicted by the achievable rates of individual hops on different OFDM tones.

In addition to the multihop relaying schemes, the joint routing and scheduling problem challenges as well the multihop cooperative schemes in relay networks. In fact, the authors in [4] observe that to devise efficient routing algorithms to establish a path through multiple clusters of possible cooperating nodes, the problem is in principle joint routing, clustering, and resource allocation.

It is worth mentioning that in the context of relay-enhanced cellular networks, "in-cell routing," or simply relay selection in case of two-hop relaying within the cell vicinity, is the most commonly considered [20]. Nevertheless, more sophisticated schemes that enable a sort of mesh topology exploit the substantial increase in degrees of freedom by establishing routes comprising relay nodes even located in adjacent cells, i.e., inter-cell routing. Such RRM schemes, despite incurring further complexity, are of great research interest [21].

5) CAC and Handover for Network-Load Balancing via Inter-Cell Routing: As earlier mentioned, RRM schemes have to work in conjunction with a CAC mechanism that decides, based on available resources and connected users' QoS, whether to admit an incoming connection to a particular cell (BS) or deny it and hand the user over to a neighboring noncongested cell through a handover mechanism. Such mechanisms are essential to balance the load network-wide and reduce the blocking probability. With the deployment of relays, more handover opportunities arise through enabling inter-cell routing. In that case, a user with denied connection to one cell can be admitted to an adjacent cell by establishing connection through one or more RS(s) in the latter. This dynamic load-balancing mechanism is termed "primary relaying" in [22], in which the authors integrate ad hoc relaying schemes into cellular networks. An alternative mechanism termed "secondary relaying" is also proposed, where an ongoing connection can be diverted through RSs to an adjacent cell, and the vacant resources are then inherited by the incoming connection. Such mechanism is beneficial whenever handover opportunities are limited for the user with incoming connection request due to traffic and channel conditions.

Although the concept could be generalized, to alleviate the burden on the cellular resources, these dynamic load-balancing mechanisms rely on the availability of out-of-band channels, such as the 2.4-GHz industrial, scientific, and medical band, to facilitate multihop relying and user access [23]. Several works have analyzed the performance of these mechanisms and proposed different routing or path-selection criteria; a 
mathematical theory has been developed in [24]. It has been noted, however, in [4] that out-of-band relaying requires UTs and RSs equipped with multiple radios operating on different frequency bands: the default cellular interface and an ad hoc wireless interface. Moreover, we observe that these inter-cell routing mechanisms were proposed and analyzed for time-division multiple-access/code-division multiple-access (TDMA/CDMA)-based networks rather than OFDMA-based relay networks.

\section{B. CCI in OFDMA Cellular Relay Networks}

Another burdensome challenge that faces the RRM schemes in next-generation cellular relay networks is the increase in CCI. In fact, CCI is inherent in any multicellular network mainly due to inter-cell and intra-cell resource reuse. Clustering, cell sectorization, and static/dynamic fractional frequency reuse are common techniques used to control the ICI in conventional cellular networks. With the increasing demand of high data rates in next-generation networks, highly aggressive reuse schemes are envisioned to achieve a much higher spectral efficiency. The aggressive reuse suggests that entire system resources will be made available in each cell, whereas intracell spatial reuse can be applied to further improve resource utilization given that a part is consumed in facilitating multihop relaying on orthogonal channels.

On the cell level, some techniques, such as dynamic power allocation [17], can be employed, as a link adaptive technique, to either mitigate or cope with the excessive CCI in such interference-limited systems. It is, however, observed that the vast majority of works apply static intra-cell spatial reuse patterns solely based on user locations, e.g., reusing the channels assigned to RS-UT links in the BS-UT links within the close vicinity of the BS [25], [26]. Such static reuse patterns are not informed by the resultant CCI. However, more intelligent RRM schemes are supposed to control the CCI through opportunistic intra-cell reuse utilizing instantaneous channel conditions and antenna directivity [13]. In such a case, the least CCI levels are attained, and underutilized resources, if any, are used first. Thus, different channels will have different reuse factor realizations.

More importantly, while relays are deployed with the potential of improving coverage and assisting users having unfavorable channel conditions (e.g., cell-edge users), an adverse effect arises. That is, in the downlink scenario, the relays deployed in one cell bring the interference closer to the cell-edge users in the adjacent cells; this potentially increases the level of ICI and renders a more interference-limited system for the prospective RRM schemes in their attempt to attain the desired high spectral efficiency.

Therefore, some recent works, e.g., [27] and [28], have extended the static fractional frequency reuse technique to OFDM cellular relay networks as a compromise solution between aggressive reuse and cell-edge performance due to the ICI caused by the neighboring relays. In both works, a three-sector cellular network is considered, where the fractional frequency bands are used for the RS-UT communication at the relay coverage area, whereas the whole band is used for the BS-UT and BS-RS communication in each sector. However, such techniques require planning and limit the opportunities a prospective relaybased scheme could exploit in frequency, multiuser, and spatial diversities within the relay coverage area.

Having observed that cell-edge relays potentially increase the level of ICI, an interesting question could be can relays help mitigate the ICI? In fact, some works, such as [29] and [30], do employ the cell-edge relays in mitigating the ICI. In [29], an RS located at the cell edge is shared by the surrounding BSs and is equipped with multiple antennas. The shared RS separates the received mixed signals and performs interference suppression using multiple-input-multiple-output (MIMO) techniques, on the same resource block, before forwarding to the respective UTs; we will revisit this approach in Section III. Using a different approach, the algorithm in [30] (the case study in Section VI) utilizes the existence of relays and their uniform geographical deployment to spatially randomize the ICI through its inherent cell-load balancing feature, which results in an even distribution of OFDM subchannels among the active relays of each cell. As such, a cell-edge user being served by a relay in one cell is less likely to receive ICI from the closest relay of the adjacent cell.

On the network level, dynamic inter-cell coordination could be the candidate for addressing interference problems. Dynamic inter-cell coordination can be employed by exchanging vital interference information among BSs over the backbone network connection to achieve the interference avoidance gain and improve user throughput and/or fairness. Although several inter-cell coordination schemes have been proposed in the literature for conventional and LTE cellular networks [31], [32], there have been no proposals for dynamic coordination schemes designed for cellular relay networks so far. Interestingly, our efforts toward devising dynamic inter-cell coordination schemes for OFDMA-based cellular relay networks led to some important observations on the issue of pronounced "interference uncertainty."

Challenges of CCI Uncertainty: Due to the dynamic and synchronous nature of the network-level distributed resourceallocation process in multicarrier cellular networks, the lack of interference predictability represents a challenging problem that has been observed in the literature of conventional OFDMA-based cellular networks. Generally, such uncertainty of subcarrier quality arises when the network is partially loaded such that BSs, based on individual allocation decisions, are not fully utilizing the set of available subcarriers. Various techniques that aim at increasing the predictability of interference in conventional OFDMA-based cellular networks are discussed in [5], which we summarize as follows:

1) partitioning (imposing structures): power shaping over time slots within the frame, on-off power shaping over the cell sectors in time, fixing the sequence of resource allocation to users, or limited exchange of interference information between neighboring BSs;

2) discretization of the quantities involved in the resource allocation, such as transmit power levels, steering coefficients of the directional antennas, and transmission rates. 
Although some of these techniques may still be imported to a relay-enhanced type of network, they will basically compromise the aggressiveness in resource reuse and limit the available degrees of freedom. Moreover, these techniques are anticipated to be less efficient in combating the uncertainty problem as it becomes more pronounced in relay networks. The main reason is not farfetched; the problem originates from the dramatic increase in ICI dynamics resulting from switching over different link budgets of potential interfering links as the subcarrier assignment hops among different cell nodes (see Fig. 2). In other words, a receiving node on a particular subcarrier, somewhere in the network, will experience a dramatic change in interfering signal strength when the subcarrierto-node assignment changes in the interfering cell from one allocation instant to another. To the best of our knowledge, current literature on relay-enhanced networks overlooked such deterioration in the CCI uncertainty. However, our results for RRA schemes and some dynamic inter-cell coordination schemes as applied to OFDMA cellular relay networks revealed the following.

1) Some dynamic inter-cell coordination schemes may not tolerate the increased CCI uncertainty as their embedded optimization criteria rely on the assessment of how much "harm" each individual interferer causes to other nodes before taking decisions against the most harmful interferers. As such, relying on the outdated interference power observed during the previous transmission can obviously overestimate a tolerable interferer or underestimate a very strong interferer in the following transmission. Therefore, the overall performance gain in throughput or fairness might be insignificant if not in the negative direction. For instance, this has been observed in the performance of a binary power control coordination scheme in which a central controller is employed to only resolve the conflicts in suppression requests issued by the receiving users or relays against their most harmful interferers in the adjacent cells.

2) On the other hand, RRA algorithms designed for OFDMA-based relay networks might, however, tolerate the increased uncertainty as the subcarrier quality metric used is usually tied to the sum of received interference power, e.g., signal-to-interference-plus-noise ratio (SINR), rather than individual interferer strength. Thereafter, if practical adaptive modulation and coding (AMC) is employed as a link-adaptive technique, a wide range of these SINRs is then quantized to one out of a few AMC modes or spectral efficiencies [33]. More importantly, any greedy or even fairness-aware RRA algorithm will end up allocating the "best" subcarrier through a comparative or a sorting routine. As such, the allocation result is not necessarily sensitive to the actual values of the outdated quality metrics or SINRs (observed during the previous transmission), and therefore, the algorithm might still achieve its desired objective without significant performance losses.

Since the last observed interference power values may not represent the actual interference situation when transmission

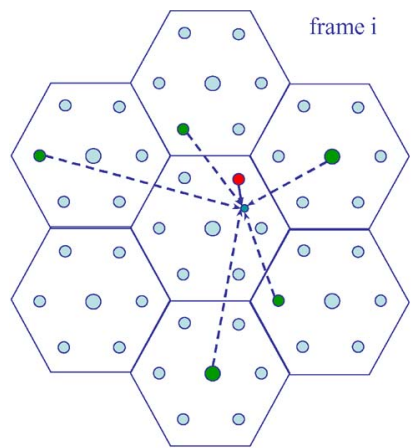

(a)

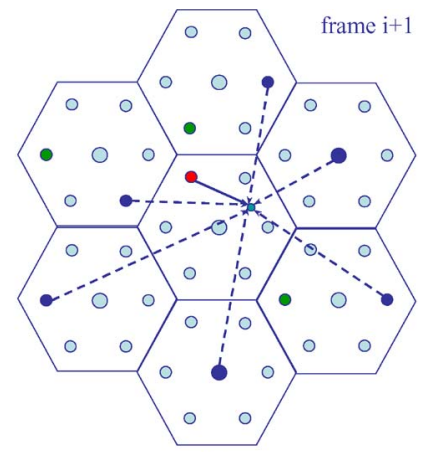

(b)
Fig. 2. Snap shots of the cellular network in downlink explaining the CCI uncertainty problem. (a) CCI during frame $i$. (b) CCI during frame $i+1$. Solid arrows represent the desired signals in the center cell.

takes place, optimization could rely on a statistical value of the past sequence of previous interference measurements. For instance, a moving-average interference filtering approach has been examined in our studies for its simplicity in terms of computation [similar to the filtering used in the proportional fair scheduler (PFS)]. This recursive single-tap filter basically provides one output sample $\tilde{I}(t)$ of interference that is more influenced by immediate past measurements than earlier measurements, within a time window controlled by $\alpha$, as

$$
\tilde{I}(t)=(1-\alpha) \tilde{I}(t-1)+\alpha I(t-1), \quad 0<\alpha<1 .
$$

The parameter $\alpha$ can carefully be chosen. A review on research development toward adapting transmission using the statistics of measured SINRs, rather than relying on the outdated information itself, is provided in [34].

\section{Restating the Strategy for Improving Goodput Efficiency}

As earlier mentioned, both dynamic inter-cell coordination and link adaptation are possible techniques for CCI avoidance or mitigation. Both techniques rely on channel state information (CSI) feedback from the receiver to the transmitter as frequent as the relevant resource-allocation process. Since the net throughput or "goodput" depends on how much feedback overhead penalty the RRM scheme incurs, the CSI feedback represents an additional challenge to any prospective centralized RRM schemes in OFDMA-based cellular relay networks. This is because, in principle, the required CSI feedback in such networks is substantially higher than that required for conventional cellular networks due to the following reasons.

1) Signals at source nodes have multiple potential recipients, among which, the routing algorithm selects the appropriate receiving nodes.

2) Relays, as receivers, are also required to provide CSI feedback to the source node(s).

3) Half-duplex relaying (HDR) is commonly employed due to current practical limitations on the operation of wireless relays. In HDR, the transmission time frame is split into two consecutive subframes (e.g., in downlink, BSs transmit, and then BSs and RSs transmit), and thus, the links that are active during both subframes experience two different interference situations that need to be 
reported within the duration of a frame, as compared with a single interference situation in the case of conventional cellular networks. In fact, this applies even if the channel gains do not change along the whole frame duration.

\section{Role of Multiantenna Systems IN THE FUTURE NETWORKS}

The combination of multiantenna systems in the form of MIMO and OFDMA has been shown to yield a rich synergy. From the RRM perspective, MIMO in its simplest implementation can significantly improve the multiplexing gain of system links. Such boost in the quality of communication links yields significant savings in system resources as fewer resource blocks are needed therein to satisfy the users' QoS requirements. Thus, system capacity in terms of the number of users and/or traffic load is increased, and a reliable service is attained. In fact, the LTE technology has positioned itself to exploit this opportunity. For conventional MIMO, different UTs are expected to carry multiple antennas according to their size constraints.

Different strategies are required to enjoy the benefit of MIMO transmission in a scenario where multiple antennas are not deployable at the terminal. For instance, cooperative relaying schemes and protocols are envisioned to build virtual antenna arrays from distributed single-antenna terminals [35]. This, however, is possible in the evolved LTE, i.e., LTEAdvanced, where relays are an integral part of the technology. The current LTE specification indicates that mixed (in terms of the number of antennas) terminals are supportable, and thus, point-to-point MIMO can be realized for terminals equipped with multiple antennas. For single-antenna terminals, the conventional maximal ratio combining technique can be used to improve the system reliability since the eNodeBs (LTE BSs) are deployed with multiple antennas.

Another important aspect of multiantenna relay-aided systems is the opportunity of establishing efficient multihop routes through beamforming and the smart-antenna capabilities of nulling the CCI from neighboring users or relays. A more elaborate exploitation of the combined relaying and multiantenna technologies is observed in [29], where multiantenna cell-edge relays are shared by the surrounding BSs and separate the received mixed signals using MIMO techniques. Without resorting to resource planning and partitioning, such a scheme alleviates the interference burden on system resources and further facilitates aggressive reuse.

In summary, multiantenna systems will provide a great opportunity for advanced signal processing (such as beamforming, precoding, and multiplexing, among others), which are essential for reliable and bandwidth-efficient system deployment.

\section{Extending CONVENTIONAL SCHEDUling Algorithms to CEllular RElay Networks}

A common strategy to extend a conventional non-relaying scheduler to relay networks, though not optimal, is the generic framework of partitioning the users into clusters around the chosen serving nodes (BS and RSs), and based on this partitioning, resources are shared among the nodes. Each node then schedules the users as in conventional OFDMA systems. Examples are the partial proportional fair (PPF) scheduler and the extended/greedy round-robin schedulers in [36] and the reference scheme in [37].

The PFS is a widely used scheduler that provides a compromise solution between network-capacity-greedy scheduling and user-fairness-oriented scheduling. Thus, it realizes the multiuser diversity gains to some extent while maintaining a degree of fairness across UTs [38]. A simple implementation of this scheduler allocates a resource unit to the UT that maximizes the ratio of its achievable rate on that unit to its exponentially weighted average rate [39].

Although PFS is known in the literature to provide an efficient throughput-fairness tradeoff, incorporating this scheduler in multicarrier systems has not vigorously been investigated [40]. This is going to change soon given the recent developments in the literature. Through the strategy described earlier, some heuristics have integrated PFS into combined relay and OFDMA technologies, such as in [26], [41], and [42]. Since the $\mathrm{BS}$ node is required to allocate the resources among the direct UTs and the feeder links of the RSs, a priority metric for such feeders to contend with direct UTs has been proposed in [41]. For the relay-enhanced scheme proposed in [42], a potential improvement in proportional fairness sense can be realized through the clustering (in-cell routing) criterion of UTs, which aims as well at maximizing the proportional fairness metric. PFS has also been implemented in [43] considering both halfand full-duplex UTs in a multihop frequency-division duplexing (FDD) network. However, due mainly to the inherent lack of queue awareness in the PFS besides the suboptimal extension strategy, the relay-enhanced scheme proposed in [19] is shown to provide more fairness in the throughput rate sense.

In addition to the prominent challenges, it is important to point out the fairness opportunities residing in such rich environment with numerous degrees of freedom. In the following section, we discuss various fairness types, fairness-assessment methods, as well as some possible fairness implementations in prospective RRA algorithms.

\section{FAIRnESS IN ORTHOGONAL FREQUENCY-DIVISION Multiple ACCESS Relay Networks: An Obligation or A Privilege}

Schedulers in OFDMA-based networks can be designed to fully exploit multiuser and channel diversities in both time and frequency to maximize the total cell capacity in a greedy manner at the expense of fairness among users. Such a design approach is network centric and does not take the individual users' QoS into account. Thus, the applicability of these algorithms in practical cellular networks is doubtful since users pertaining to the same service class will similarly be charged while the service is not evenly distributed. Clearly, from a roaming user's perspective, the inability to maintain fairness defeats service reliability and ubiquity as it becomes channel and location dependent. That would be deemed a failure on the part of the service provider, despite the technology and ingenuity embedded in network equipment. In that sense, fairness as an obligation rather than a privilege ensures user satisfaction, regardless of location and channel conditions. 
Therefore, user fairness expectations would be even higher in modern relay networks, where service providers advertise outstanding QoS based on the new architecture widely adopted by the state-of-the-art standards. With less diversity sacrifices in mind, relaying promises ubiquitous coverage and QoS improvement for users with unfavorable channel conditions. Fairness obligation is thus stronger, whereas keeping such promises is conditioned on the awareness and smartness of the RRM scheme in use.

Relative fairness [44] is a very important aspect when it comes to practical implementation. That is because users subscribe to different service classes offered by the service provider for different costs per bit. Thus, heterogeneous traffic flows are expected to coexist with different statistical nature and QoS requirements. Therefore, fairness assessment has to be conducted within subgroups of users that pertain to the same service classes taking into account their respective on and off traffic bursts. Otherwise, to incorporate multiple classes into the fairness assessment, some works have proposed modified fairness metrics or assessment criterion by introducing relative weights [45] or using normalized throughput [46], [47]. In that sense, results reflect on the relative fairness yet they may vary from a time window to another within a session due to the different traffic properties.

Various fairness classes are noted in [48], such as shortterm fairness, long-term fairness, and time-average fairness. The classification is dependent on the time window size used to evaluate the chosen fairness metric. Generally, achieving shortterm fairness imposes stringent constraints on the RRM scheme while less-stringent constraints for the long-term fairness and relaxed constraints for the time-average fairness. The choice of the appropriate fairness class to investigate the fairness of an RRM scheme should be based on the traffic model and the relevant QoS requirements.

Relay fairness is another important aspect of RRM in OFDMA-based relay networks, which is different from user fairness because there are no QoS requirements specific to RSs. In fact, relay fairness, as shown in the literature, aims at distributing the traffic load almost evenly among RSs so that no RS will be overloaded [7]. In [49], relay fairness is assessed based on the power consumption at the relays, under different relay-selection mechanisms, to operate a cooperative diversity scheme without overloading the battery of one or more relay(s). We also note that if the relay's transmit power per subcarrier is fixed, maintaining almost even distribution of subcarriers among relays limits the relay's total transmit power and, thus, its power-amplifier rating and the consumption of its battery energy for battery-operated relays. In addition, a balanced traffic load reduces the packet processing delays at the regenerative relays. Therefore, we observe that the term "cellload balancing," as earlier defined in Section II, and the term "relay fairness" can be used interchangeably, regardless of the measure used to quantify the load.

\section{A. Fairness Metric Examples}

Fairness functions can be viewed from two different perspectives: fairness criteria implemented in routing and scheduling algorithms to assess priorities of users and evaluate whether compensation is required and fairness metrics used to investigate and classify fairness-aware algorithms. In the following, we briefly mention the most commonly used fairness functions or metrics, whereas some numerical examples are shown through the case study in Section VI.

1) The proportional fairness metric: Considering a network with $K$ users with the same service class and priority, the scheme that maximizes the proportional fairness metric as

$$
F=\sum_{i=1}^{K} \log r_{i}
$$

is fairer according to the game-theoretic definition of proportional fairness, which implies that any change in the "proportionally fair" rate allocation $\boldsymbol{x}$ to, for example, rate allocation $\boldsymbol{y}$, must have a negative total relative change [38], i.e., $\Sigma_{i=1}^{K}\left(r_{i}^{y}-r_{i}^{x} / r_{i}^{x}\right) \leq 0$.

2) Jain's fairness index [50] assumes a value between $1 / K$ and unity for a network having $K$ users with the same service class and priority. Mathematically, Jain's index can be expressed as

$$
x=\frac{\left(\sum_{i=1}^{K} r_{i}\right)^{2}}{K \sum_{i=1}^{K} r_{i}^{2}} .
$$

3) The IEEE 802.16m fairness index [51] maps each user's rate to a value between 0 and $K$ as expressed in the following:

$$
x_{j}=\frac{r_{j}}{\frac{1}{K} \sum_{i=1}^{K} r_{i}} .
$$

Numerical examples are shown through the case study in Section VI.

4) The fairness factor [48] has been developed to measure fairness in RRM noncooperative static games. As shown in (5), this factor represents the normalized statistical standard deviation of user's throughput compared with that of a single-user case. In contrast with Jain's index, the higher the factor, the less the fairness realized among users, i.e.,

$$
\rho=\frac{1}{\bar{T}} \sqrt{\frac{1}{K-1} \sum_{i=1}^{K}\left(\frac{T_{i}}{T_{i}^{\max }}-\bar{T}\right)^{2}}
$$

where $T_{i}^{\max }$ is the maximum throughput user $i$ could solely achieve while $\bar{T}=$ average $\left(\bar{T}_{i}\right), \bar{T}_{i}=$ average $\left(T_{i} / T_{i}^{\max }\right)$.

\section{B. Throughput-Fairness Tradeoff in OFDMA Relay Networks}

The tradeoff between aggregate network throughput and user fairness has vigorously been investigated in the literature of scheduling in conventional cellular networks. The concept refers to the tendency of the scheduling algorithm to sacrifice, to some extent, the throughput opportunities provided by the multiuser/channel diversity to attain a degree of user fairness. 
However, it is worth noting that with burst traffic, such a tradeoff may not always exist. For instance, a queue-aware scheduler would improve fairness without incurring a throughput penalty when it schedules some users with low channel qualities and occupied buffers after scheduling the queues of the user(s) with the best channels exhaustively.

Among the conventional networks, multiuser OFDM- and OFDMA-based networks generally offer better opportunity for schedulers to exploit the multiuser and channel diversities in both time and frequency domains and achieve a more efficient throughput-fairness tradeoff [52], e.g., the algorithm in [53]. Further enhancing OFDMA-based networks with relays has remarkably enriched the wireless environment through the reduced path loss, the increased spatial diversity, and the performance-boosting multihop-relaying and cooperativediversity schemes. As such, fewer diversity sacrifices are expected to attain the desired user fairness in OFDMA-based relay networks; compared with conventional networks, user fairness should not cost the system performance as much sacrifice in terms of throughput opportunities, and significantly more efficient tradeoffs can potentially be achieved. However, as noted earlier in Section V, achieving such an efficient tradeoff is conditioned on the awareness and smartness of the RRM scheme in use. That also implies that an intelligent RRM scheme may outperform other poorly designed schemes, i.e., with inefficient tradeoffs, in both throughput and user fairness and not necessarily in one aspect on the expense of the other. This could be the case as well if comparing the network performance, possibly under the same scheduling policy, with and without relay assistance. These observations have been captured by the performance results of the schemes in [30] and [54], which are presented in the case study in Section VI.

In fact, some works in the literature of OFDMA relay networks have demonstrated the difference in performance and the ability to exploit the available diversities between some basic scheduling approaches as extended to relay networks using strategies similar to that described in Section IV. Among these extended basic approaches are the round-robin, the PFS, and the Max-SINR, which have been shown in [26] to respectively possess greater capacity greediness. In addition to these approaches, the extended PPF and greedy polling schedulers are compared in [36]. The tradeoff has been addressed as well in [41], where a fairer scheduler, namely, two-hop proportional fair, attains significantly less aggregate throughput.

\section{Fairness Implementation in RRA Algorithms}

Basic scheduling approaches and criterion (e.g., max-min, round-robin or proportional fairness), as well as popular practical schedulers such as the channel-state-dependent packet scheduling, the channel-independent packet fair queueing, and the server-based fair approach, were proposed for the conventional networks. So far, there are no provisions, other than that discussed in Section IV, for how such approaches should be applied to a relay-enhanced cellular network, which is different from the conventional downlink point-to-multipoint problem. Therefore, new algorithms need to be developed to facilitate fair scheduling and routing in such networks. While the literature in this area is steadily growing, one can realize that most of the algorithms proposed so far focus on total capacity maximization and, sometimes, with some fairness constraints imposed on the optimization problem, e.g., [6] and [55].

Since capacity does not directly map to throughput due to traffic burstness, such scheduling and routing algorithms will not satisfy the QoS requirements because they are unaware of traffic and queues status. In other words, allocating resources for some links solely based on SINRs or achievable rates, even under fairness constraints, can result in substantial performance losses if no sufficient data reside at the buffers of source nodes at that allocation instant. For that reason, full queues at all potential source nodes [25] are often implied in some studies, e.g., [15].

An interesting approach therefore is to involve buffer states or queue lengths in the formulation of the optimization problem, and given that the allocation process is conducted in a relatively fast manner, no prior knowledge of the traffic-arrival processes is required. As such, not only is a waste of resources avoided, but also, "traffic diversity" is exploited, which means that when some users' traffic is in the off period, more resources can be utilized to provide a better and fairer service to the other users.

One way of involving the buffer states in the formulation is to work out the optimization problem as a sum-utility or sumdemand maximization, where, for instance, such a metric could be a stochastic drift that is proportional to both the difference in queue length between the source and destination buffers and the link quality at the destination. Such a scheduling policy belongs to the class of throughput-optimal link-scheduling policies inspired by the theory in [18] and developed in the context of multihop packet-radio networks. Several throughputoptimal RRA algorithms have been proposed thereafter for non-OFDMA and/or non-relaying networks with different optimization problems. In [56] and [57], modulated versions of this metric are used in conventional cellular Space Division Multiple Access/TDMA and OFDMA networks, respectively. Generally, queue awareness allows RRM schemes to take corrective actions in following allocation frames to compensate the overlooked user buffers, if any, and potentially improve fairness among homogenous traffic flows, at least in the long term. However, these corrective actions are limited to subchannel allocation in conventional RRM schemes, which clearly cannot combat large path loss unless handover between neighboring BSs is employed. This, however, has implications on load balancing among BSs. In contrast, queue-aware relaying schemes offer the opportunity to circumvent the problem of large path loss, for instance, due to heavy shadowing, through in-cell routing. This is done by selecting the appropriate RS(s) and resource units for the associated hops.

It should be noted that throughput-optimal policies are not fairness oriented in principle, as they aim to stabilize all user queues under any heterogeneous traffic flows within the system's capacity region. Therefore, in [58], for instance, a congestion-control mechanism is proposed with such policy employed to introduce user fairness through traffic policing if the arrival rates are elastic, i.e., the traffic sources can adapt their rates. 


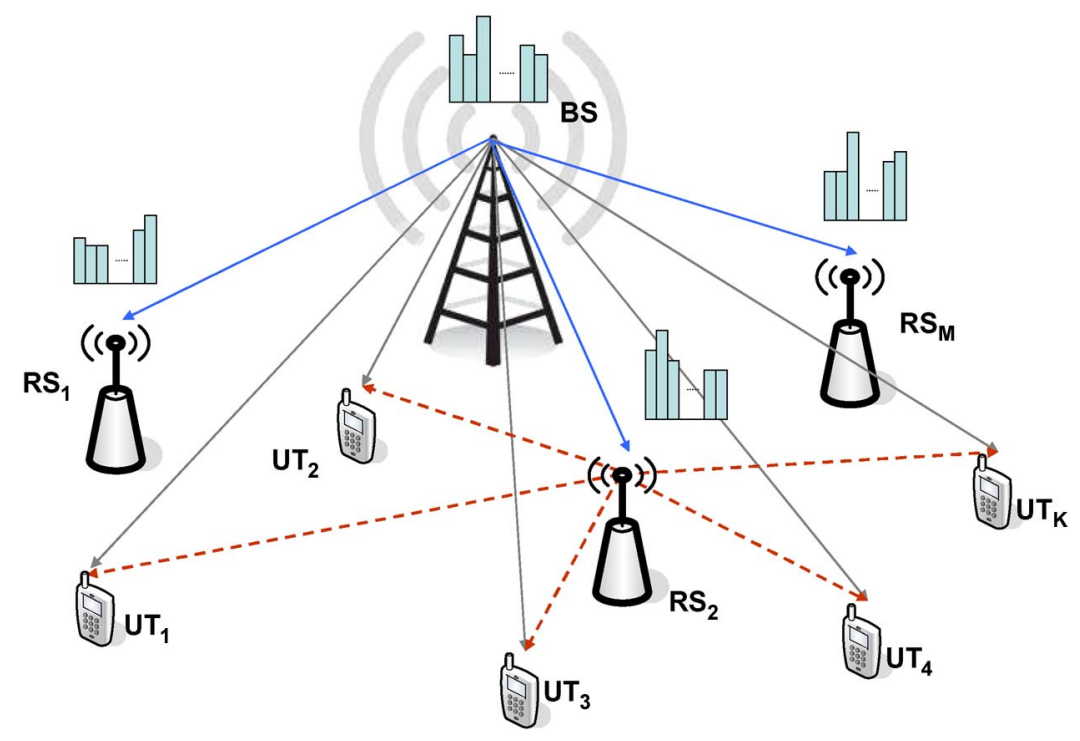

Fig. 3. Example partial network of BS and relays showing a snap shot of user queues and the potential links of the BS and $\mathrm{RS}_{2}$ on subchannel $n$.

In the next section, a joint-routing and fair-scheduling algorithm that employs a similar policy is presented. The algorithm represents a different optimization problem in an OFDMAbased relay-enhanced network [30]. The objective is to maximize the total cell throughput while maintaining throughput fairness among users. The idea is to operate a throughputoptimal scheduling policy that stabilizes user queues at all nodes in a system that receives homogenous inelastic traffic processes at only one source node in the cell, which is the BS. Therefore, the fair behavior of such policy is a special case due to the considered cellular system model, where all users belong to the same service class and, thus, have the same mean arrival rates and the same QoS requirements. Such a policy is also perceived fair, given a similar scenario in [16] but in a relayenhanced single-carrier CDMA network.

\section{Illustrative CASE Study}

We emphasize that the purpose of the case study presented in this section is to illustrate and contextualize most of the topics addressed in this survey paper. The discussion is based on the material presented in [30]. Therefore, in the subsequent discussions, we employ the style in this reference. The downlink of a multicellular network is considered, where each cell has $K$ UTs. These UTs are served through the BS directly or through any of the cell's $M$ RSs. The system may operate in either time-division duplexing (TDD) or FDD modes. All cells use the same spectrum, resulting in a unity reuse factor. Through sectorization, higher reuse is attainable by employing the same scheme in each sector, e.g., LTE-A architecture. The total bandwidth is divided into $N$ subchannels each consisting of a set of adjacent OFDM data subcarriers. According to the previous discussion in Section II, the scheme is network-level distributed/cell-level centralized.

The CSI is available at the transmitters in the form of persubchannel SINRs or the corresponding achievable rates taking into account the ICI and assuming that AMC is employed. However, substantial savings in feedback overhead are achieved by only reporting the index of the achievable AMC level [30]. The serving BS and each of the $M$ relays in a cell are equipped with $K$ user buffers. User packets arrive at the corresponding BS buffer according to the UT's service class and traffic model (same for all users). The total transmit power available at any node is equally divided among its allocated subchannels. A UT can be receiving from a group of nodes, and any node can transmit to multiple destinations on different orthogonal subchannels. Also, any RS in that scheme is assumed to have the ability to concurrently receive and transmit different data on orthogonal subchannels during downlink (DL) or uplink (UL). This duplexing mode, such as in [15] and [42], is termed quasi full-duplex relaying (QFDR), which is different from the theoretical full-duplex mode considered earlier in the literature [59], [60]. The practical implications on self interference from adjacent subchannels are discussed in [30].

Nevertheless, the scheme can be modified for HDR operation such that the downlink resource allocation is split into two consecutive allocation processes: 1) a BS subframe followed by 2 ) an RS subframe. This new optimization problem considering HDR and based on the same joint routing and scheduling policy is presented in [54], where further improvements in terms of system's capacity and latency have been achieved. We also note that the term "HDR relaying" has been used differently in [61] to describe a relaying protocol for half-duplex UTs in an FDD system.

\section{A. Joint-Routing and Fair-Scheduling Algorithm}

Fig. 3 shows a part of the multicellular network to demonstrate the basic principle of operation of the representative RRM in a relay-enhanced OFDMA-based network. In the uplink, relays update the $\mathrm{BS}$ (node 0) with the actual queue size information for only the previously relayed UTs. $Q_{k}^{m}$ is the queue length of UT $k$ at node $m$ in bits, bytes, or equal-size packets (shown in blue bars in Fig. 3). A UT is the sink for its respective data flow and, thus, has zero queue length. At any allocation instant, only one link is active per subchannel. 
Let us first define the demand metric for each type of links: For any user-access link node $m-\mathrm{UT}_{k}$, the demand metric on subchannel $n$ is calculated as

$$
D_{n, m \rightarrow k}=R_{m, k, n} Q_{k}^{m}, \quad m=0,1, \ldots, M
$$

where $R_{m, k, n}$ is the achievable rate (or spectral efficiency) on the link node ${ }_{m}-\mathrm{UT}_{k}$ based on the SINR $\gamma_{m, k, n}$ calculated using the filtering approach ${ }^{1}$ mentioned in Section II. As the BS has the choice to use a subchannel on a direct user access link or on a relay feeder link, the demand of any BS-RS ${ }_{m}$ link on subchannel $n$, which has an achievable rate $R_{0, m, n}$, is defined as in (6) and incorporates the maximum differential backlog of the queues of the BS and those of $\mathrm{RS}_{m}$ [16], [17] as

$$
D_{n, 0 \rightarrow m}=R_{0, m, n} \max _{k}\left\{\left(Q_{k}^{0}-Q_{k}^{m}\right)^{+}\right\}
$$

where $(\cdot)^{+}$sets negative values to 0 . Note that if the feeder link BS-RS ${ }_{m}$ is assigned subchannel $n$, then the data flow that is nominated by this maximum differential backlog is scheduled; routing is thus jointly performed with scheduling.

To maximize the total cell throughput while stabilizing user queues at all nodes, the RRA scheme needs to optimize the assignment of subchannels to all user access links and to all feeder links with their selected data flows so that the sum demand is maximized at each allocation instant. The resource allocation at the BS can therefore be formulated as a 3-D assignment problem that falls in the category of binary integer linear programming (BILP) under some constraints, which ensure that at most one link is active per subchannel, guarantee even distribution of subchannels among all nodes and, hence, achieves cell-load balancing, and finally ensure efficient bit loading of subchannels to avoid resource wastage. However, the computational complexity of a brute-force solution to this 3-D BILP problem is prohibitive and nonpolynomial in time and can be approximated to $\mathcal{O}\left(((M+1) K)^{N}\right)$. Therefore, a nearoptimal low-complexity algorithm is devised with an upper polynomial bound of $\mathcal{O}\left(N^{2}(N+M+1)^{2} / 4(M+1)\right)$.

Utilizing the absence of interdependency between the potential links at each subchannel node pair $(n, m)$, the link with the maximum demand at each pair is nominated without compromising the optimal solution. Since usually $N>M$, the algorithm iteratively solves the problem such that in each iteration a 2-D one-to-one assignment problem is optimally solved using the Hungarian algorithm [62]. The buffer states are updated and the assigned subchannels are eliminated between iterations while the aforementioned constraints are satisfied. Hence, the algorithm can be executed at the beginning of each allocation time frame through the following steps:

1) The demand metric of $\mathrm{RS}_{m}$ on subchannel $n$ is calculated as the maximum of $K$ potential links as follows:

$$
D_{n, m}=\max _{k}\left\{R_{m, k, n} Q_{k}^{m}\right\}, \quad m=1,2, \ldots, M .
$$

\footnotetext{
${ }^{1}$ A marginal performance gain has been observed, as compared with employing the SINR values of the previous DL frame due to the uncertainty problem discussed earlier.
}

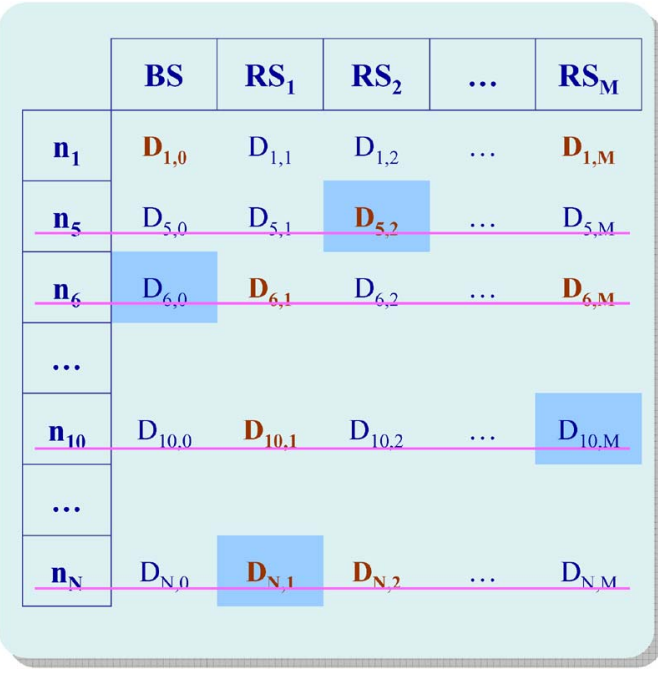

Fig. 4. Demand matrix during one iteration. Rows with assigned entries are crossed out and eliminated. Bold red entries reflect on the queue updates due to the previous iteration.

Thus, $D_{n, m}$ is the best proposal of $\mathrm{RS}_{m}$ to use subchannel $n$, whereas the UT associated with that maximum is marked as the candidate receiver. The demand metric for the BS node is the maximum metric of $M+K$ potential links and is expressed as

$$
D_{n, 0}=\max _{j}\left\{D_{n, 0 \rightarrow j}\right\}
$$

where $D_{n, 0 \rightarrow j}$ is calculated using (6) and (7), and $j$ denotes any of the $M+K$ potential destinations. Thus, $D_{n, 0}$ is the best proposal of the BS to use subchannel $n$. The destination associated with that proposal is marked as the candidate receiver. Note that if the destination is an RS, then the UT that achieved the maximum queue difference on that link is marked as well.

2) After calculating the $(M+1)$ demand metrics on each subchannel, the algorithm solves a one-to-one optimization problem to maximize the total demand by applying the Hungarian algorithm to the $N \times(M+1)$ demand matrix $\left[D_{n, m}\right]$ (see Fig. 4).

3) The algorithm virtually updates the affected UTs' queues according to the decisions of the previous iteration as

$$
Q_{k}^{m^{(\imath+1)}}=\left(Q_{k}^{m^{(\imath)}}-\left\lfloor R_{m}^{(\imath)} T\right\rfloor\right)^{+} .
$$

$Q_{k}^{m^{(\imath)}}$ is the input queue length to iteration $\imath$, and $R_{m}^{(\imath)}$ is the rate of the link assigned by the Hungarian algorithm to node $m$ as a result of iteration $\imath$. Note that the queues at destination RSs are not incremented between iterations because the transmissions on all subchannels simultaneously occur in the QFDR mode, and the algorithm has to obey the causality law.

4) The rows corresponding to the assigned subchannels are eliminated.

5) Steps 1)-4) are repeated for the unassigned subchannels until all enqueued packets are scheduled or the subchannels are exhausted. 
Due to the one-to-one assignment, each iteration will only assign $M+1$ subchannels to the $M+1$ nodes. As a result, each node is linked to only one destination per iteration; this prevents, along with step 3 , the scheduling errors that could happen when the same queue length is involved in the choice of more than one link. Furthermore, if $N \bmod (M+1)=0$, then each node will be assigned exactly $N /(M+1)$ subchannels. It therefore implies that load balancing or relay fairness is inherent in that algorithm. During any iteration, if a node has exactly zero demand values on all subchannels, then it is excluded by default from that Hungarian iteration.

Routing of user packets from the BS is dependent on the second-hop link quality, as the following example shows: Let us assume that $\mathrm{RS}_{M}$ in Fig. 3 has a very poor link to $\mathrm{UT}_{3}$ (e.g., due to heavy shadowing), whereas the BS has forwarded some $\mathrm{UT}_{3}$ packets to $\mathrm{RS}_{M}$ (as the user with the maximum differential backlog therein). In this situation, these packets might not be forwarded to $\mathrm{UT}_{3}$. This is not an error; the algorithm exploits the presence of these trapped packets as they reflect on the quality of the second-hop link to $\mathrm{UT}_{3}$. That is because in the next allocation frame, the BS will identify the user $k^{*}$ that has the maximum difference $Q_{k}^{0}-Q_{k}^{M}$ as the candidate user on that feeder link who is unlikely to be $\mathrm{UT}_{3}$. Hence, the trapped packets reduce the likelihood of routing $\mathrm{UT}_{3}$ data again through that link on any of the subchannels, whereas other user queues at $\mathrm{RS}_{M}$ are being discharged from one iteration to another. Thus, the probability of forwarding $\mathrm{UT}_{3}$ packets to $\mathrm{RS}_{M}$ very rapidly converges to zero, particularly if the cell has a high density of UTs and RSs. The algorithm therefore possesses a learning ability, as subsequently observed. ${ }^{2}$

Results: The described algorithm is evaluated through comprehensive system-level simulations using the WiMAX parameters [63] based on the IEEE 802.16e and $802.16 \mathrm{~m}$. The WINNER ${ }^{3}$ C2 LOS channel model is employed for the BS-RS links. We hereby mention some essential parameters to provide insight into the simulation environment. The network consists of 19 hexagonal cells. The distance between two adjacent BSs is $1 \mathrm{~km}$. Relays are placed at a distance of 0.65 of the cell radius from the BS and with a uniform angular spacing. The BS-RS links experience 4-dB lognormal shadowing and time-frequency correlated Rician fading with 10-dB Rician factor. All the other links experience 8.9-dB independent lognormal shadowing and time-frequency correlated Rayleigh fading. The UTs are dropped from a uniform distribution on the cell area. Shadowing and UT location are fixed during a drop. A bandwidth of $20 \mathrm{MHz}$ is divided into 102 subchannels. The TDD frame length is $2 \mathrm{~ms}$ with a $2: 1$ downlink-to-uplink ratio. The transmit powers for BSs and RSs are 46 and $37 \mathrm{dBm}$, respectively. Independent Poisson packet arrival processes are assumed at BS queues with an average arrival rate of 632 packets (188 B each) per second per UT. For more details, see [30].

The statistics of time-average user throughput are studied through the cumulative distribution function (CDF) plots shown

\footnotetext{
${ }^{2}$ More elaborate discussions on the algorithm's optimality, convergence, and learning ability of this dynamic routing strategy are contained in [19].

${ }^{3}$ EU's Sixth Framework Project, IST WINNER: www.ist-winner.org.
}

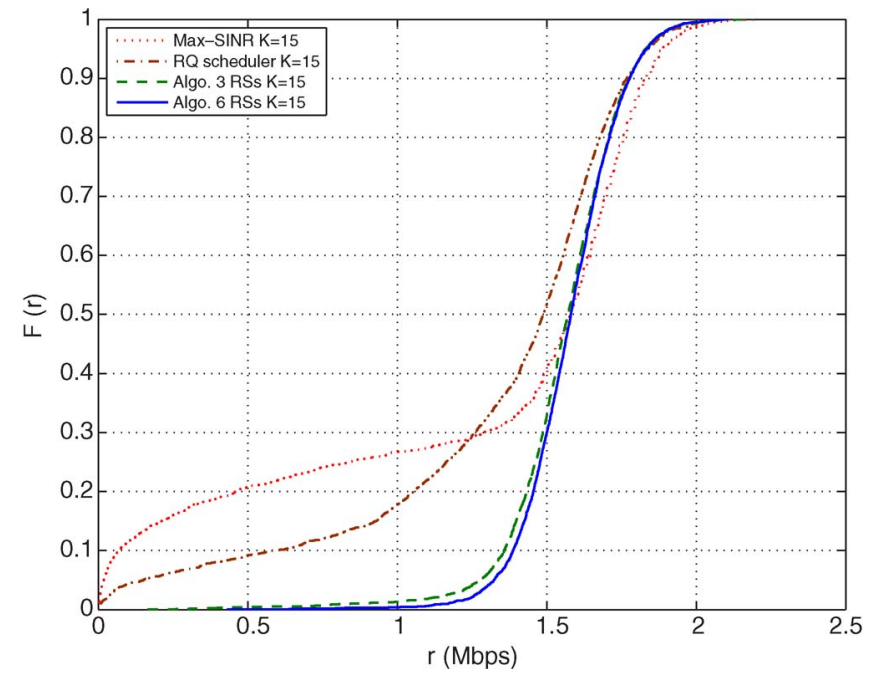

Fig. 5. CDF of time-average user throughput at 15 users/cell.

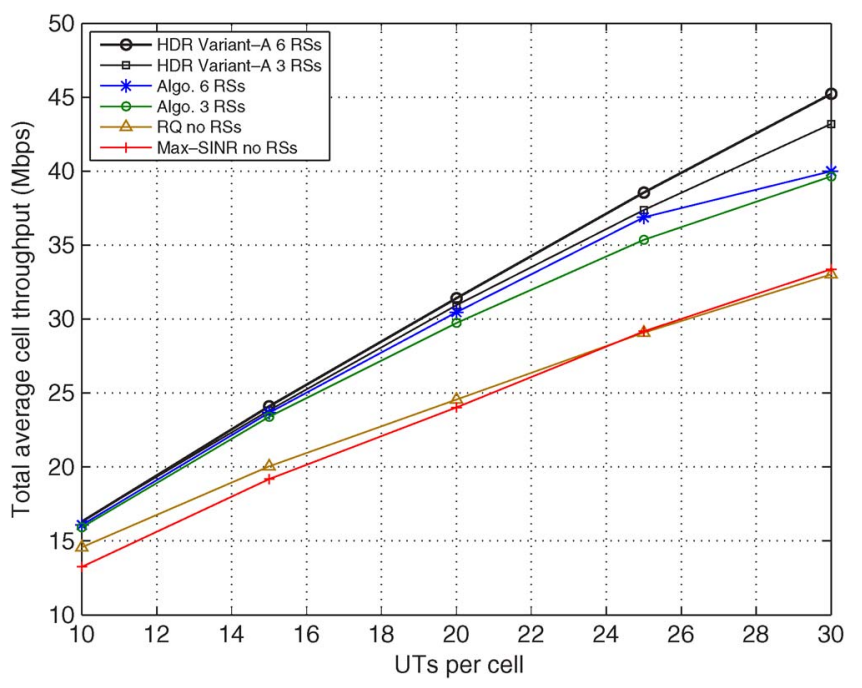

Fig. 6. System's capacity in terms of number of UTs/cell at the given mean arrival rate for the algorithm with three and six RSs and the reference conventional schedulers. Higher capacity is attained through the HDR scheme in [54] based on the same policy.

in Fig. 5 for 15 users/cell. The fifth percentile throughput of the CDFs is associated with cell-edge users in LTE evaluation methodology [64]. A significant five percentile throughput advantage is realized for the no-relay queue-aware R-times-Q (RQ) scheduler as compared with the no-relay Max-SINR scheduler, ${ }^{4}$ which achieves a corresponding throughput of almost zero with a bad lower tail behavior. Substantial throughput improvements of $730 \%$ and $760 \%$ are realized with respect to the "RQ" scheduler for the relaying scheme with three and six RSs, respectively. As an alternative interpretation, at a target average throughput of $1 \mathrm{Mb} / \mathrm{s}$, the outage probability is $0.4 \%$ in the case of six RSs, and it is $1.4 \%$ in the case of three RSs, as compared with $18 \%$ for the RQ scheduler and $27 \%$ for the Max-SINR.

The behavior of the curves in Fig. 6 is in agreement with the multiuser diversity concept and emphasizes the multihop

\footnotetext{
${ }^{4}$ It assigns a subchannel to the best user of only those with buffered data
} 


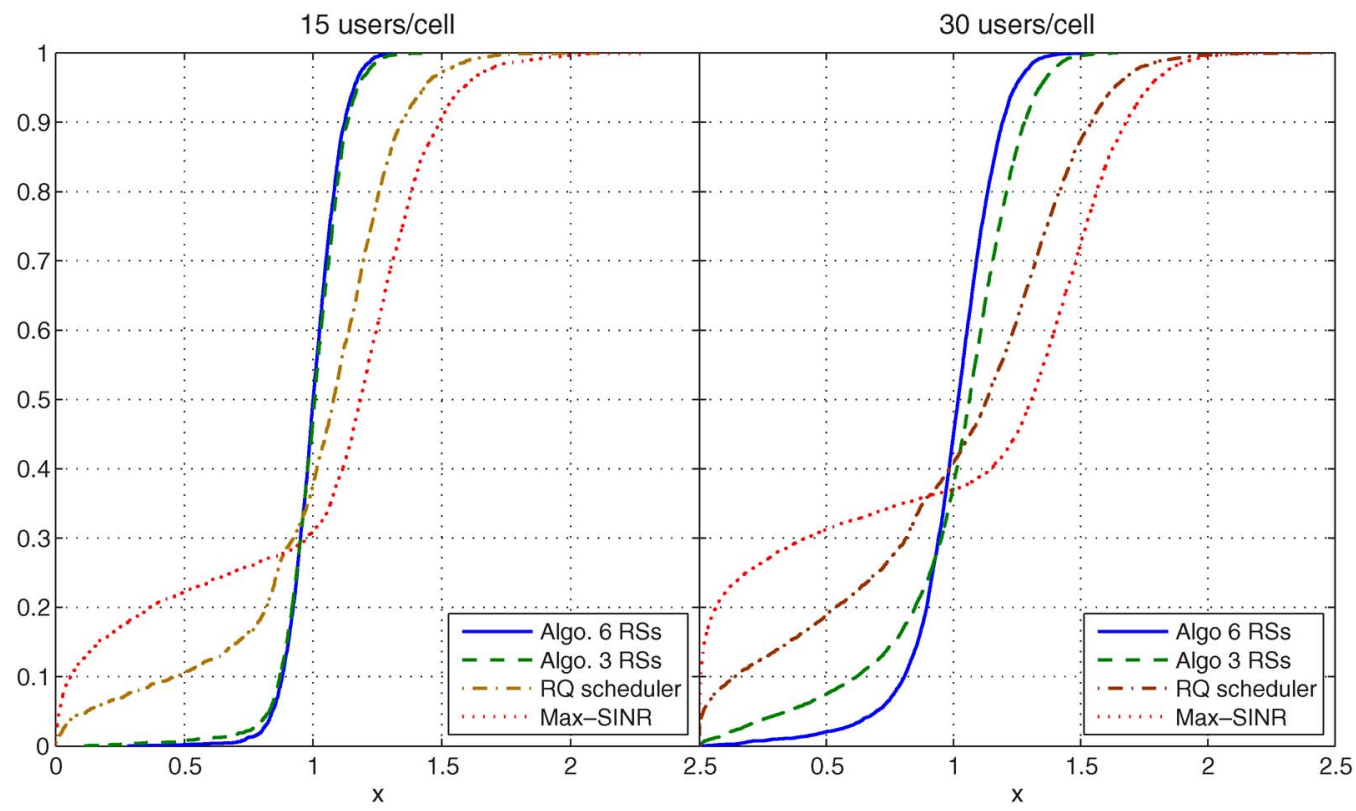

Fig. 7. Time-average fairness through the CDF of the IEEE $802.16 \mathrm{~m}$ index for a multicell network with 15 and 30 users/cell.

relaying gain compared with the same policy without relays (the RQ scheduler). The aggregate traffic load is increased in terms of the number of UTs per cell. It can be observed that given the same amount of system resources, the capacity of the system has significantly been increased through relay assistance using the joint algorithm, which exploits the opportunities in such environment, at low to moderate loadings. At higher loads, the slope indicates a performance bottleneck as compared with the HDR variant (A) devised in [54] to improve the capacity, latency, and practicality of the system based on the same scheduling policy across two consecutive subframes of the DL frame.

While the time-average fairness can be realized from the trend in the previous CDFs, the IEEE $802.16 \mathrm{~m}$ fairness index and Jain's fairness index are employed to further assess the performance in terms of time-average and long-term fairness, respectively. The values of the $802.16 \mathrm{~m}$ index are collected based on the users' time-average throughput from each drop to plot the CDFs shown in Fig. 7 for 15 and 30 users/cell. Recall that the closer the CDF plot is to a step function at unity, the fairer the scheme is to the UTs pertaining to the same service class. Although the RQ scheduler is a form of throughputoptimal scheduling, we observe a significant time-average fairness advantage with respect to the Max-SINR scheduler that is mainly due to the fact that the RQ scheduler allows none of the symmetric and equal-priority user flows to be overlooked, unlike the greedy Max-SINR scheduler, which may not even map the allocated capacity to throughput due to the lack of queue-size awareness. The best fairness behavior is achieved when the relaying scheme manages the RSs to provide a fair service for all users. This is evident from the difference between the scenarios with three and six RSs under different loading conditions.

As a matter of fact, long-term fairness is a more stringent fairness assessment than time-average fairness. In Fig. 8, the closer the CDF to a unit step at unity, the more long-term

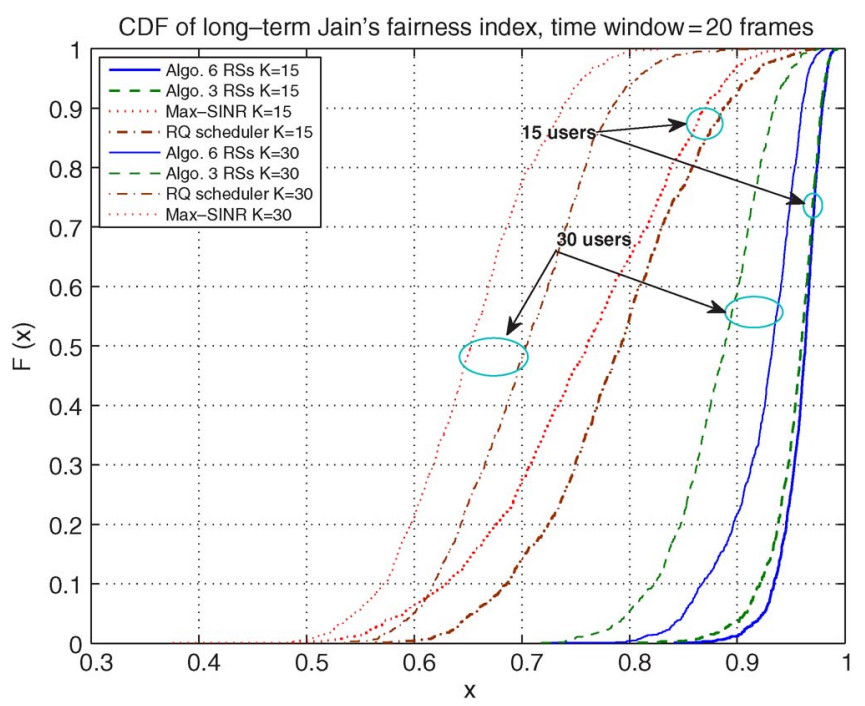

Fig. 8. Long-term fairness over 20 time frames through Jain's fairness index for a multicell network with 15 and $30 \mathrm{UTs} / \mathrm{cell}$ and the assistance of three or six RSs for the relaying algorithm.

fairness the scheme achieves over a time window of 20 frames. A substantial improvement in long-term fairness is realized through the relaying scheme, as compared with the reference schemes, including the queue-aware RQ scheduler, regardless of the different shadowing and path loss experienced in each drop. That is, in fact, a good example on the fairness opportunities in routing in relay-enhanced networks, as discussed in Section V-C. Fig. 9 shows the time-average throughput as a function of shadowing and user distance from the BS using a scatter plot. The plot is obtained by time averaging the throughput for each UT within a drop with fixed location and shadowing. The scatter plots are approximated by thirddegree polynomials using least-square curve fitting as a means of averaging over shadowing and thus represent the distancebased conditional mean. For the joint routing and scheduling 


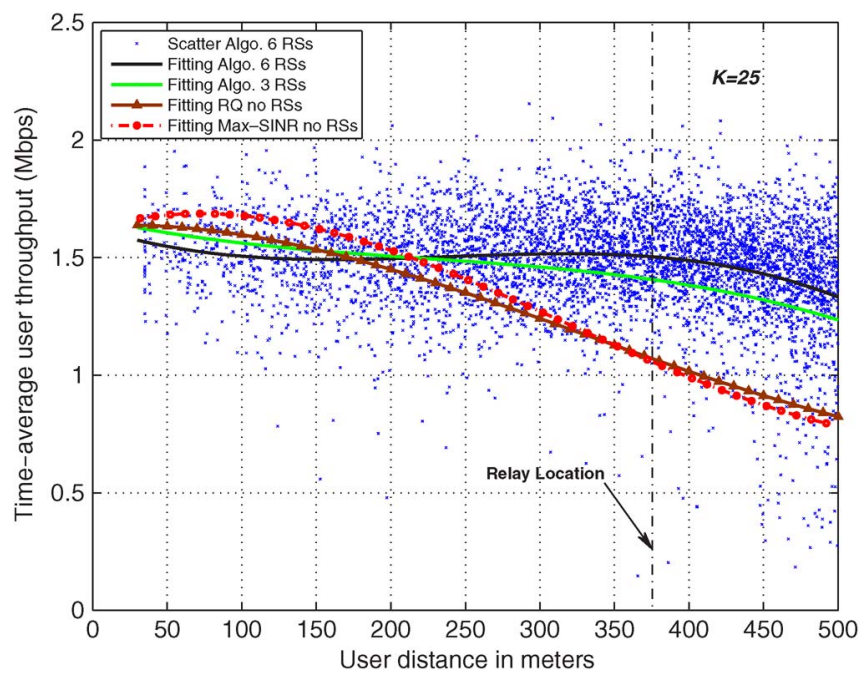

Fig. 9. Service ubiquity and cell-edge performance. Scatter plot of the timeaverage user throughput as a function of shadowing and user distance from the BS at $25 \mathrm{UTs} /$ cell. Distance-based conditional mean is presented by the curve fitting.

algorithm, the uniform average throughput across the cell area is clearly evident from the almost-flat performance from the $\mathrm{BS}$ to the cell edge. This implies that a fair service and ubiquitous coverage are provided for all users, regardless of their location, channel, and interference conditions. Unlike the reference conventional schedulers, which cannot combat large path losses, it can be observed that the user throughput at the cell edge is comparable with that of the users close to the BS under that relaying scheme. Note that the Max-SINR scheduler implemented here is often forced to serve some cell-edge users whenever the buffers of the best users are vacant; the cell-edge performance therefore severely degrades at higher traffic loads.

Moreover, relay fairness has also been jointly achieved through the inherent load-balancing feature. Histograms of the number of assigned subchannels to each node have been provided in [30] and attest to such behavior. Recall that the load-balancing feature utilizes the uniform geographical relay deployment in spatially spreading the ICI, which counteracts the increased interference levels due to the proximity of RSs to the cell-edge UTs of adjacent cells and aggressive resource reuse.

\section{CONCLUSION}

Relaying techniques and OFDMA technologies are envisioned to provide ubiquitous high-data-rate coverage and reliable service for next-generation wireless networks. Devising intelligent RRM schemes is crucial to harness the opportunities in such future OFDMA-based relay-enhanced networks, where conventional schemes are not adequate. This paper has addressed some of the opportunities, challenges, and terms associated with the migration from conventional cellular to relay-enhanced architecture. Users' expectations are much higher, given the advertised objectives, and fairness obligation is stronger in such networks. This paper has thus discussed some fairness-implementation techniques along with some exemplary fairness metrics toward the design and performance evaluation of prospective fair RRA algorithms. While the literature in this area is steadily growing, this paper has introduced a case study where a joint routing and fairscheduling algorithm has shown significant fairness behavior and achieved ubiquitous coverage. The example algorithm has also achieved relay fairness and ICI mitigation through its inherent cell-load balancing capability.

\section{REFERENCES}

[1] R. Pabst, B. Walke, D. Schultz, P. Herhold, H. Yanikomeroglu, S. Mukherjee, H. Viswanathan, M. Lott, W. Zirwas, M. Dohler, H. Aghvami, D. Falconer, and G. Fettweis, "Relay-based deployment concepts for wireless and mobile broadband cellular radio," IEEE Commun. Mag., vol. 42, no. 9, pp. 80-89, Sep. 2004.

[2] E. Bertin, I. Fodil, and N. Crespi, "A business view for NGN service usage," in Proc. IEEE/IFIP Int. Workshop Broadband Convergence Netw. May 2007, pp. 1-5.

[3] J. Bae, S. Kang, J. Kim, B. H. Park, K. Kim, and G. Sihn, "Provision of user-centric mobile services in next generation mobile communication environment," in Proc. IEEE Veh. Technol. Conf., Sep. 2007, pp. 2005-2009.

[4] L. Le and E. Hossain, "Multihop cellular networks: Potential gains, research challenges, and a resource allocation framework," IEEE Commun. Mag., vol. 45, no. 9, pp. 66-73, Sep. 2007.

[5] D. Gesbert, S. Kiani, A. Gendhemsjo, and G. Oien, "Adaptation, coordination, and distributed resource allocation in interference-limited wireless networks," Proc. IEEE, vol. 95, no. 12, pp. 2393-2409, Dec. 2007.

[6] C. Bae and D.-H. Cho, "Fairness-aware adaptive resource allocation scheme in multihop OFDMA systems," IEEE Commun. Lett., vol. 11, no. 2, pp. 134-136, Feb. 2007.

[7] G. Li and H. Liu, "Resource allocation for OFDMA relay networks with fairness constraints," IEEE J. Sel. Areas Commun., vol. 24, no. 11, pp. 2061-2069, Nov. 2006.

[8] J. Lee, H. Wang, S. Lim, and D. Hong, "A multi-hop user admission algorithm for fixed relay stations with limited capabilities in OFDMA cellular networks," in Proc. IEEE 18th Int. Symp. Pers., Indoor, Mobile Radio Commun., Sep. 2007, pp. 1-5.

[9] T. Kim, T.-Y. Min, and C. Kang, "Opportunistic packet scheduling algorithm for load balancing in a multi-hop relay-enhanced cellular OFDMA-TDD system," in Proc. IEEE 14th Asia-Pacific Conf. Commun., Oct. 2008, pp. 1-5.

[10] Mobile Multihop Relay Study Group Recommendations for the Scope and Purpose of the Mobile Multihop Relay Task Group, IEEE Std. 802.16, Nov. 11, 2005. [Online]. Available: http:www.ieee802.org/16/sg/mmr/ contrib/C80216mmr-05_032.pdf

[11] Draft IEEE Standard for Local and Metropolitan Area Networks Part 16: Air Interface for Fixed and Mobile Broadband Wireless Access Systems: Multihop Relay Specification, IEEE Std. P802.16j/D1, pp. 1002-1007, Aug. 2007

[12] D. Soldani and S. Dixit, "Wireless relays for broadband access," IEEE Commun. Mag., vol. 46, no. 3, pp. 58-66, Mar. 2008.

[13] M. Salem, A. Adinoyi, H. Yanikomeroglu, and Y.-D. Kim, "Radio resource management in OFDMA-based cellular networks enhanced with fixed and nomadic relays," in Proc. IEEE Wireless Commun. Netw. Conf., Sydney, Australia, Apr. 2010, pp. 18-21.

[14] M. Salem, A. Adinoyi, H. Yanikomeroglu, and Y.-D. Kim, "Nomadic relay-directed joint power and subchannel allocation in OFDMA-based cellular fixed relay networks," in Proc. IEEE Veh. Technol. Conf., Taipei, Taiwan, May 2010, pp. 16-19.

[15] J. Lee, S. Park, H. Wang, and D. Hong, "QoS-guarantee transmission scheme selection for OFDMA multi-hop cellular networks," in Proc, IEEE Int. Conf. Commun., Jun. 2007, pp. 4587-4591.

[16] H. Viswanathan and S. Mukherjee, "Performance of cellular networks with relays and centralized scheduling," IEEE Trans. Wireless Commun., vol. 4, no. 5, pp. 2318-2328, Sep. 2005.

[17] M. Neely, E. Modiano, and C. E. Rohrs, "Dynamic power allocation and routing for time-varying wireless networks," IEEE J. Sel. Areas Commun., vol. 23, no. 1, pp. 89-103, Jan. 2005.

[18] L. Tassiulas and A. Ephremides, "Stability properties of constrained queueing systems and scheduling policies for maximum throughput in multihop radio networks," IEEE Trans. Autom. Control, vol. 18, no. 6, pp. 1936-1948, Dec. 1992. 
[19] M. Salem, A. Adinoyi, M. Rahman, H. Yanikomeroglu, D. Falconer, Y.-D. Kim, W. Shin, and E. Kim, "Fairness-aware radio resource management in downlink OFDMA cellular relay networks," IEEE Trans. Wireless Commun., 2010.

[20] V. Sreng, H. Yanikomeroglu, and D. Falconer, "Relayer selection strategies in cellular networks with peer-to-peer relaying," in Proc. IEEE Veh. Technol. Conf., Oct. 2003, pp. 1949-1953.

[21] J. Shi, Z. Zhang, P. Qiu, and G. Yu, "Subcarrier and power allocation for OFDMA-based regenerative multi-hop links," in Proc. Int. Conf. Wireless Commun., Netw., Mobile Comput., Sep. 2005, pp. 207-210.

[22] H. Wu, C. Qiao, S. De, and O. Tonguz, "Integrated cellular and ad-hoc relay systems: iCAR," IEEE J. Sel. Areas Commun., vol. 19, no. 10, pp. 2105-2115, Oct. 2001.

[23] E. Yanmaz and O. Tonguz, "Dynamic load balancing and sharing performance of integrated wireless networks," IEEE J. Sel. Areas Commun., vol. 22, no. 5, pp. 862-872, Jun. 2004.

[24] E. Yanmaz and O. Tonguz, "The mathematical theory of dynamic load balancing in cellular networks," IEEE Trans. Mobile Comput., vol. 7, no. 12, pp. 1504-1518, Dec. 2008.

[25] M. Kaneko and P. Popovski, "Radio resource allocation algorithm for relay-aided cellular OFDMA system," in Proc. IEEE Int. Conf. Commun., Jun. 2007, pp. 4831-4836.

[26] Ö. Oyman, "Opportunistic scheduling and spectrum reuse in relay-based cellular OFDMA networks," in Proc. IEEE Global Commun. Conf., Nov. 2007, pp. 3699-3703.

[27] Y. Zhao, X. Fang, X. Hu, Z. Zhao, and Y. Long, "Fractional frequency reuse schemes and performance evaluation for OFDMA multi-hop cellular networks," in Proc. Int. Conf. Testbeds Res. Infrastructures Develop. Netw. Commun. Workshops, Apr. 2009, pp. 1-5.

[28] W. Lee, M.-V. Nguyen, J. Jeong, B. Keum, and H. Lee, "An orthogonal resource allocation algorithm to improve the performance of OFDMAbased cellular wireless systems using relays," in Proc. IEEE Consumer Commun. Netw. Conf., Jan. 2008, pp. 917-921.

[29] Y. Song, H. Yang, J. Liu, L. Cai, D. Li, X. Zhu, K. Wu, and H. Liu, "Relay station shared by multiple base stations for inter-cell interference mitigation,” IEEE Std. 802.16 Broadband Wireless Access Working Group, IEEE C80216m-08/1436, Oct. 2008.

[30] M. Salem, A. Adinoyi, M. Rahman, H. Yanikomeroglu, D. Falconer, Y.-D. Kim, W. Shin, and E. Kim, "Fairness-aware joint routing and scheduling in OFDMA-based cellular fixed relay networks," in Proc. IEEE Int. Conf. Commun., Jun. 2009, pp. 1-6.

[31] M. Rahman and H. Yanikomeroglu, "Interference avoidance through dynamic downlink OFDMA subchannel allocation using intercell coordination," in Proc. IEEE Veh. Technol. Conf., May 2008, pp. 1630-1635.

[32] M. Rahman, H. Yanikomeroglu, and W. Wong, "Interference avoidance with dynamic inter-cell coordination for downlink LTE systems," in Proc. IEEE Wireless Commun. Netw. Conf., Apr. 2009, pp. 1238-1243.

[33] A. J. Goldsmith and S. G. Chua, "Adaptive coded modulation for fading channels," IEEE Trans. Commun., vol. 46, no. 5, pp. 595-602, May 1998.

[34] H. Zhang, S. Wei, G. Ananthaswamy, and D. L. Goeckel, "Adaptive signaling based on statistical characterizations of outdated feedback in wireless communications," Proc. IEEE, vol. 95, no. 12, pp. 2337-2353, Dec. 2007.

[35] A. Adinoyi and H. Yanikomeroglu, "Cooperative relaying in multiantenna fixed relay networks," IEEE Trans. Wireless Commun., vol. 6 , no. 2, pp. 533-544, Feb. 2007.

[36] L. Huang, M. Rong, L. Wang, Y. Xue, and E. Schulz, "Resource scheduling for OFDMA/TDD based relay enhanced cellular networks," in Proc. IEEE Wireless Commun. Netw. Conf., Mar. 2007, pp. 15441548.

[37] Z. Tang and G. Wei, "Resource allocation with fairness consideration in OFDMA-based relay networks," in Proc. IEEE Wireless Commun. Netw. Conf., Apr. 2009, pp. 1630-1634.

[38] F. Kelly, A. Maulloo, and D. Tan, "Rate control for communication networks: Shadow prices, proportional fairness and stability," J. Oper. Res. Soc., vol. 49, no. 3, pp. 237-252, Mar. 1998.

[39] P. Viswanath, D. Tse, and R. Laroia, "Opportunistic beamforming using dumb antennas," IEEE Trans. Inf. Theory, vol. 48, no. 6, pp. 1277-1294, Jun. 2002.

[40] W. Anchun, X. Liang, Z. Shidong, X. Xibin, and Y. Yan, "Dynamic resource management in the fourth generation wireless systems," in Proc. Int. Conf. Commun. Technol., Apr. 2003, pp. 1095-1098.

[41] L. Xiao and L. Cuthbert, "Improving fairness in relay-based access networks," in Proc. 11th Int. Symp. Model., Anal. Simul. Wireless Mobile Syst., Oct. 2008, pp. 18-22.
[42] W.-G. Ahn and H.-M. Kim, "Proportional fair scheduling in relay enhanced cellular OFDMA systems," in Proc. IEEE 19th Int. Symp. Pers. Indoor, Mobile Radio Commun., Sep. 2008, pp. 1-4.

[43] R. Schoenen, A. Otyakmaz, and B. Walke, "Concurrent operation of half- and full-duplex terminals in future multi-hop FDD based cellular networks," in Proc. IEEE Int. Conf. Wireless Commun., Netw., Mobile Comput., Oct. 2008, pp. 1-7.

[44] Y. Zhou and H. Sethu, "On the relationship between absolute and relative fairness bounds," IEEE Commun. Lett., vol. 6, no. 1, pp. 37-39, Jan. 2002.

[45] R. Elliott, "A measure of fairness of service for scheduling algorithms in multiuser systems," in Proc. IEEE Can. Conf. Elect. Comput. Eng., May 2002, vol. 3, pp. 1583-1588.

[46] A. V. Babu and L. Jacob, "Fairness analysis of IEEE 802.11 multirate wireless LANs," IEEE Trans. Veh. Technol., vol. 56, pt. 2, no. 5, pp. 3073 3088, Sep. 2007.

[47] F. Bokhari, H. Yanikomeroglu, W. K. Wong, and M. Rahman, "Fairness assessment of the adaptive token bank fair queuing scheduling algorithm," in Proc. IEEE Veh. Technol. Conf., Sep. 2008, pp, $1-5$.

[48] Z. Han and K. J. R. Liu, Resource Allocation for Wireless Networks: Basics, Techniques, and Applications. Cambridge, MA: Cambridge Univ. Press, 2008.

[49] J. Vicario, A. Bel, A. Morell, and G. Seco-Granados, "Outage probability versus fairness trade-off in opportunistic relay selection with outdated CSI," EURASIP J. Wireless Commun. Netw., vol. 2009, p. 9, Jan. 2009.

[50] R. Jain, The Art of Computer Systems Performance Analysis: Techniques for Experimental Design, Measurement, Simulation and Modeling. New York: Wiley, 1991.

[51] IEEE Std. 802.16 Broadband Wireless Access Working Group, IEEE C802.16m-07/306 Protocol structure to support cooperative transmission. [Online]. Available: http://www.ieee802.org/16/tgm/contrib/C80216m07_306.pdf

[52] S. Sadr, A. Anpalagan, and K. Raahemifar, "Radio resource allocation algorithms for the downlink of multiuser OFDM communication systems," Commun. Surveys Tuts., vol. 11, no. 3, pp. 92-106, 3rd Quar., 2009.

[53] Z. Shen, J. Andrews, and B. Evans, "Adaptive resource allocation in multiuser OFDM systems with proportional rate constraints," IEEE Trans. Wireless Commun., vol. 4, no. 6, pp. 2726-2737, Nov. 2005.

[54] M. Salem, A. Adinoyi, H. Yanikomeroglu, D. Falconer, and Y.-D. Kim, "A fair radio resource allocation scheme for ubiquitous high-data-rate coverage in OFDMA-based cellular relay networks," in Proc. IEEE Global Commun. Conf., Dec. 2009, pp. 1-6.

[55] W. Nam, W. Chang, S.-Y. Chung, and Y. Lee, "Transmit optimization for relay-based cellular OFDMA systems," in Proc. IEEE Int. Conf. Commun., Jun. 2007, pp. 5714-5719.

[56] M. Kobayashi and G. Caire, "Joint beamforming and scheduling for a multi-antenna downlink with imperfect transmitter channel knowledge," IEEE J. Sel. Areas Commun., vol. 25, no. 7, pp. 1468-1477, Sep. 2007.

[57] P. Parag, S. Bhashyam, and R. Aravind, "A subcarrier allocation algorithm for OFDMA using buffer and channel state information," in Proc. IEEE Veh. Technol. Conf., Sep. 2005, pp. 622-625.

[58] A. Eryilmaz and R. Srikant, "Fair resource allocation in wireless networks using queue-length-based scheduling and congestion control," IEEE/ACM Trans. Netw., vol. 15, no. 6, pp. 1333-1344, Dec. 2007.

[59] G. Kramer, M. Gastpar, and P. Gupta, "Cooperative strategies and capacity theorems for relay networks," IEEE Trans. Inf. Theory, vol. 51, no. 9, pp. 3037-3063, Sep. 2005.

[60] A. Høst-Madsen and J. Zhang, "Capacity bounds and power allocation for wireless relay channels," IEEE Trans. Inf. Theory, vol. 51, no. 6, pp. 2020-2040, Jun. 2005.

[61] R. Schoenen, A. Otyakmaz, and Z. Xu, "Resource allocation and scheduling in FDD multihop cellular systems," in Proc. 2nd Int. Workshop Multiple Access Commun., ICC, Jun. 2009, pp. 1-6.

[62] H. W. Kuhn, "The Hungarian method for the assignment problem," Naval Res. Logist. Q., vol. 2, no. 1, pp. 83-97, 1955.

[63] WIMAX Forum, A comparative analysis of mobile WiMAX deployment alternatives in the access network, pp. 1-47, May 2007.

[64] E. Dahlman, H. Ekstrom, A. Furuskar, Y. Jading, J. Karlsson, M. Lundevall, and S. Parkvall, "The 3G long-term evolution-Radio interface concepts and performance evaluation," in Proc. IEEE Veh. Technol. Conf., May 2006, pp. 137-141. 


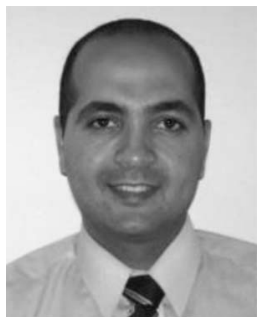

Mohamed Salem (S'06) received the B.Sc. degree in communications and electronics and the M.Sc. degree from Alexandria University, Alexandria, Egypt, in 2000 and 2006, respectively. He is currently working toward the Ph.D. degree with the Department of Systems and Computer Engineering, Carleton University, Ottawa, ON, Canada.

In February 2006, he was nominated and hired as a faculty member with the Department of Engineering Mathematics, Alexandria University, where he was promoted to the position of Assistant Lecturer. He has gained a wide range of experience in research and development and collaboration with industrial parties. He has been conducting research in collaboration with Samsung Electronics on advanced radio resource management in next-generation wireless networks. His research interests encompass stochastic modeling, congestion control, and optimization techniques.

Mr. Salem has been granted the 2009/2010 Ontario Graduate Scholarship in Science and Technology.

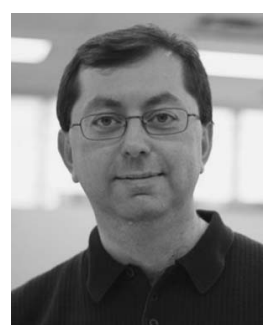

Halim Yanikomeroglu (S'97-M'98) received the B.Sc. degree in electrical and electronics engineering from the Middle East Technical University, Ankara, Turkey, in 1990 and the M.A.Sc. degree in electrical engineering (now ECE) and the Ph.D. degree in electrical and computer engineering from the University of Toronto, Toronto, ON, Canada, in 1992 and 1998, respectively.

From 1993 to 1994, he was with the R\&D Group, Marconi Kominikasyon A.S., Ankara. Since 1998, he has been with the Department of Systems and Computer Engineering, Carleton University, Ottawa, ON, Canada, where he is currently an Associate Professor with tenure. He is an Adjunct Professor with the Prince Sultan Advanced Technologies Research Institute, King Saud University, Riyadh, Saudi Arabia. He was a Guest Editor for the Wiley Journal on Wireless Communications and Mobile Computing. His research interests cover many aspects of the physical, medium-access, and networking layers of wireless communications with a special emphasis on multihop/relay/mesh networks and cooperative communications. His research is currently funded by Samsung (SAIT, Korea), Huawei (China), the Communications Research Centre of Canada (CRC), Research in Motion (Canada), and the National Science and Engineering Research Council of Canada (NSERC).

Dr. Yanikomeroglu was the recipient of the 2009 Carleton University Research Achievement Award. He has been involved in the steering committees and technical program committees of numerous international conferences. $\mathrm{He}$ has also given 18 tutorials at such conferences. He is a member of the Steering Committee of the IEEE Wireless Communications and Networking Conference (WCNC) and has been involved in the organization of this conference over many years, including serving as the Technical Program CoChair of WCNC 2004 and the Technical Program Chair of WCNC 2008. $\mathrm{He}$ is the General Co-Chair of the IEEE Vehicular Technology Conference to be held in Ottawa in September 2010. He was an Editor for the IEEE TRANSACTIONS ON WiRELESS COMMUNiCATIONS (2002-2005) and the IEEE COMMUNiCATIONS SuRveys AND Tutorials (2002-2003). He was an Officer of IEEE's Technical Committee on Personal Communications (Chair: 2005-06, Vice-Chair: 2003-04, and Secretary: 2001-02) and was a member of the IEEE Communications Society's Technical Activities Council $(2005-06)$. He is a registered Professional Engineer in the province of Ontario.

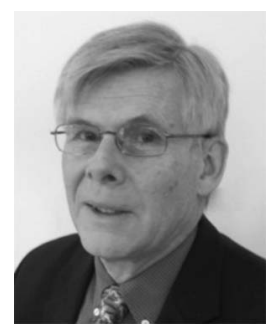

David Falconer (LF'06) received the B.A.Sc. degree in engineering physics from the University of Toronto, Toronto, ON, Canada, in 1962, the S.M. and $\mathrm{Ph} . \mathrm{D}$. degrees in electrical engineering from the Massachusetts Institute of Technology, Cambridge, in 1963 and 1967, respectively, and an honorary doctorate of science degree from the University of Edinburgh, Edinburgh, U.K., in 2009.

After a year as a postdoctoral fellow with the Royal Institute of Technology, Stockholm, Sweden, he was a member of technical staff and Group Supervisor with Bell Laboratories from 1967 to 1980. From 1976 to 1977, he was a Visiting Professor with Linköping University, Linköping, Sweden. Since 1980, he has been with Carleton University, Ottawa, ON, Canada, where he is currently Professor Emeritus and Distinguished Research Professor with the Department of Systems and Computer Engineering. He was the Director of Carleton's Broadband Communications and Wireless Systems Centre from 2000 to 2004. He was the Chair of Working Group 4 (New Radio Interfaces, Relay-Based Systems, and Smart Antennas) of the Wireless World Research Forum in 2004 and 2005. His current research interests center around beyond-third-generation broadband wireless communication systems.

Dr. Falconer received the 2008 Canadian Award for Telecommunications Research, the 2008 IEEE Technical Committee for Wireless Communications Recognition Award, and the 2009 IEEE Canada Fessenden Award (Telecommunications). 